\title{
Intervención educativa en cuidadores para prevenir úlceras por presión en adultos con inmovilidad prolongada
}

\author{
Morales-Castillejos, Lizbeth "; Austria-Pelcastre, Sandy Trinidad 2; Quevedo-Rojas, Dulce María 3; \\ Hernández-Ramírez, Mileydi 4; Galicia-Aguilar, Rosa María 5; Landeros-Olvera, Erick ${ }^{6}$
}

\section{RESUMEN}

Introducción: El conocimiento de los cuidadores acerca de la prevención de úlceras por presión en pacientes con inmovilidad es útil para evitar la aparición de lesiones, mediante la planificación, implementación y evaluación de un programa educativo en el domicilio. Objetivo: Determinar la eficacia de la intervención educativa sobre los conocimientos en la prevención de úlceras por presión en los cuidadores primarios de pacientes con inmovilidad prolongada. Metodología: diseño cuasi-experimental, muestreo aleatorio simple, se consideró al grupo de intervención como su propio control bajo un tratamiento estadístico test, re-test, con intervención simple ciego a 15 cuidadores de pacientes adultos, hospitalizados con indicación de alta y con riesgo de padecer úlceras por presión. La intervención se implementó en tres fases en cuatro sesiones, que se impartieron como taller, con una duración de treinta minutos. El análisis de los datos fue descriptivo, para comparar el test y re-test, se aplicó una t de Student. Se consideraron aspectos éticos para un estudio de bajo riesgo.

Resultados: En la fase diagnóstica el $80 \%$ de los cuidadores no tenían conocimientos, en el re-test en el 100\% los incrementó, con efecto positivo y significativo $(p=.000)$. Conclusión: El resultado obtenido muestra eficacia estadísticamente significativa de las cuatro sesiones que conformaron el programa educativo para aumentar el nivel de conocimiento de los cuidadores para la prevención de úlceras por presión.

Palabras clave: Úlcera por presión; cuidador; prevención; aprendizaje (DeCS).

\footnotetext{
${ }^{1-4}$ Estudiantes de Maestría en Enfermería. Benemérita Universidad Autónoma de Puebla (FE- BUAP)

${ }^{5}$ Maestra en Ciencias de Enfermería. (FE- BUAP)

${ }^{6}$ Doctor en Ciencias de Enfermería. (FE- BUAP)

5,6. Grupo de investigación: Intervenciones educativas para el cuidado de la salud.
}

Recibido: 15/05/2019

Aceptado: $12 / 12 / 2019$

* Autor para correspondencia: ORCID: https://orcid.org/0000-0002-3826-0508 Correo electrónico: lizbeth.moralesca@alumno.buap.mx

\section{Cómo citar este artículo}

Morales-Castillejos L, Austria-Pelcastre ST, Quevedo-Rojas DM, Hernández-Ramírez M, Galicia-Aguilar RM, Landeros-Olvera E. Intervención educativa en cuidadores para prevenir úlceras por presión en adultos con inmovilidad prolongada. SANUS. 2019; (12):6-16. [Acceso_____]; Disponible en: mes día año URL 


\title{
Intervenção educacional em cuidadores para evitar úlceras de pressão em adultos com imobilidade prolongada
}

\begin{abstract}
ABSTRATO
Introdução: O conhecimento dos cuidadores sobre a prevenção de úlceras por pressão em pacientes com imobilidades é útil para prevenir o aparecimento de lesões, por meio do planejamento, implementação e avaliação de um programa educacional em casa. Objetivo: Determinar a eficiência da intervenção educacional sobre o conhecimento sobre prevenção de úlceras por pressão em cuidadores primários de pacientes com imobilidades prolongada. Metodologia: Um delineamento quase-experimental e amostragem aleatória simples, o grupo de intervenção foi considerado como seu próprio controle sob um tratamento estatístico de teste e retorno, com intervenção cega simples a 15 cuidadores de pacientes adultos, internados como indicação de alta e em risco de sofrer úlcera por pressão. A intervenção foi implementada em três fases de quatro sessões realizadas como um workshop como duração de trinta minutos. A análise dos dados foi descritiva e, para comparar o teste e retestar, foi aplicado o teste t de Student. Questões éticas foram consideradas para uma pesquisa de baixo risco. Resultados: Na fase de diagnóstico, 80\% dos cuidadores não tinham conhecimento algum, no re-teste, 100\% deles aumentaram o conhecimento com um efeito positivo e significativo $(p=.000)$. Conclusões: $O$ resultado obtido mostrou eficiência estatisticamente significante das quatro sessões que compuseram o programa educacional para aumentar o nível de conhecimento dos cuidadores para a prevenção of úlcera por pressão.
\end{abstract}

Palavras chave: Úlcera por pressão; cuidador; prevenção; aprendendo (DeCS).

\section{INTRODUCCIÓN}

La inmovilidad prolongada, que se deriva de cualquier problema que altere la salud de la persona, es un factor condicionante en el desarrollo de úlceras por presión (UPP). Estas se definen como lesiones de origen isquémico localizadas en la piel y tejidos subyacentes que se produce por presión prolongada o fricción entre dos planos duros (1). La etiología de las UPP es multifactorial y está relacionada con el deterioro de la movilidad física, edades extremas, enfermedades crónicas, falta de higiene, presión, cizallamiento y fricción (2). Las UPP representan un importante problema de salud pública, que afecta no solo a la persona, sino a los cuidadores y al sistema de salud, debido al desarrollo de complicaciones tales como infecciones, dolor, dependencia de cuidado y aumento en los índices de morbimortalidad ${ }^{(3)}$.
De acuerdo con la OMS, a nivel internacional la prevalencia oscila entre el 5 y 12\%, en América Latina es de $7 \%$. Resultados similares se encontraron en otros países como Alemania, Brasil y España. En México en un estudio con 13,137 pacientes hospitalizados en unidades de segundo y tercer nivel en 32 estados de la república mexicana, reportaron una prevalencia media nacional de 20.07\% (4).

La evidencia científica refiere que las acciones preventivas reducen de manera significativa el desarrollo de UPP en personas que egresan del hospital, en este sentido, los profesionales de enfermería deben asumir el reto de la prevención a través la planificación, ejecución y seguimiento de cuidados dirigido a los cuidadores, que hacen referencia a las personas adultas que asumen de manera voluntaria el cuidado en el domicilio del paciente con inmovilidad prolongada para prevenir lesiones en la piel (5). Por ello, los cuidadores deben asumir esta responsabilidad, con 
conocimientos y habilidades para la valoración y prevención de las UPP en pacientes con deterioro de la movilidad física (6). Algunos autores ponen de manifiesto que la Educación para la salud (Eps) dirigida a cuidadores es una herramienta de atención primaria fundamental que tiene influencia en la eficacia para evitar la aparición de lesiones, fortalece el aprendizaje significativo, mediante estrategias que logren la autonomía e independencia en el cuidado otorgado cuando el paciente se encuentre fuera del hospital ${ }^{(7-9)}$. De acuerdo con la evidencia científica la Eps, debe fundamentarse en herramientas formativas que motiven y empoderen a los cuidadores en la adquisición de conocimientos sobre la valoración de riesgo y la implementación de medidas preventivas como la movilización, manejo de la presión, posiciones específicas y superficies de apoyo, para dar respuesta al desafío de la atención domiciliaria de la población en riesgo de sufrir UPP (10-12). Sin embargo, estudios previos en cuidadores, reportan bajo nivel de conocimientos por lo que surge el siguiente objetivo: determinar la eficacia de la intervención educativa sobre los conocimientos en la prevención de úlceras por presión en los cuidadores primarios de pacientes con inmovilidad prolongada.

\section{METODOLOGÍA}

Se realizó un diseño cuasi-experimental, con un muestreo aleatorio simple, donde se consideró al grupo de intervención como su propio control, bajo un tratamiento estadístico test, re-test, con intervención simple ciego, dirigido a 15 cuidadores primarios de pacientes adultos hospitalizados en un tercer nivel de atención. Se incluyeron pacientes de ambos géneros, hospitalizados, con indicación de alta y con riesgo de padecer úlceras por presión de acuerdo con la escala de Braden, sin considerar el diagnóstico médico.

La intervención se realizó bajo un formato simple ciego y se fundamentó en el Modelo PRECEDE (13). Este modelo se resume en tres fases. 1. Fase diagnostica: En la que se midió el nivel de conocimientos de los cuidadores primarios en la prevención de las UPP, a través de la aplicación del cuestionario COCU-LCRD 23, que reporta confiabilidad previa aceptable en población mexicana ${ }^{(14)}$, está constituido por tres apartados: el primero contiene datos del cuidador primario, el segundo datos del paciente y el tercero contiene 23 ítems que indagan los conocimientos que tiene el cuidador acerca de las UPP. El nivel de conocimientos se obtiene de la suma de un punto para las respuestas correctas y de cero para las incorrectas, donde a mayor puntuación mejor nivel de conocimientos. El análisis de los datos, se realizó a través del paquete estadístico SPSS versión 24. El análisis descriptivo se efectuó mediante frecuencias y porcentajes de las variables sociodemográficas de los cuidadores; para determinar el nivel de comparación entre el test y el re-test y se aplicó una prueba t de Student para muestras relacionadas (15). Este estudio se apegó al código de ética postulado en la declaración de Helsinki y fueron considerados los aspectos éticos para un estudio de bajo riesgo de acuerdo con la ley general de salud en materia de investigación (16).

2. Fase de planeación e implementación de la intervención. El programa educativo se desarrolló bajo la perspectiva del aprendizaje social que se caracteriza por que cada uno de los participantes comparte la responsabilidad de lograr el éxito en las actividades de aprendizaje ${ }^{(17)}$. La intervención estuvo conformada por cuatro sesiones, cada una con una duración de 30 minutos, se llevaron a cabo una vez por semana, durante seis meses. Las sesiones se

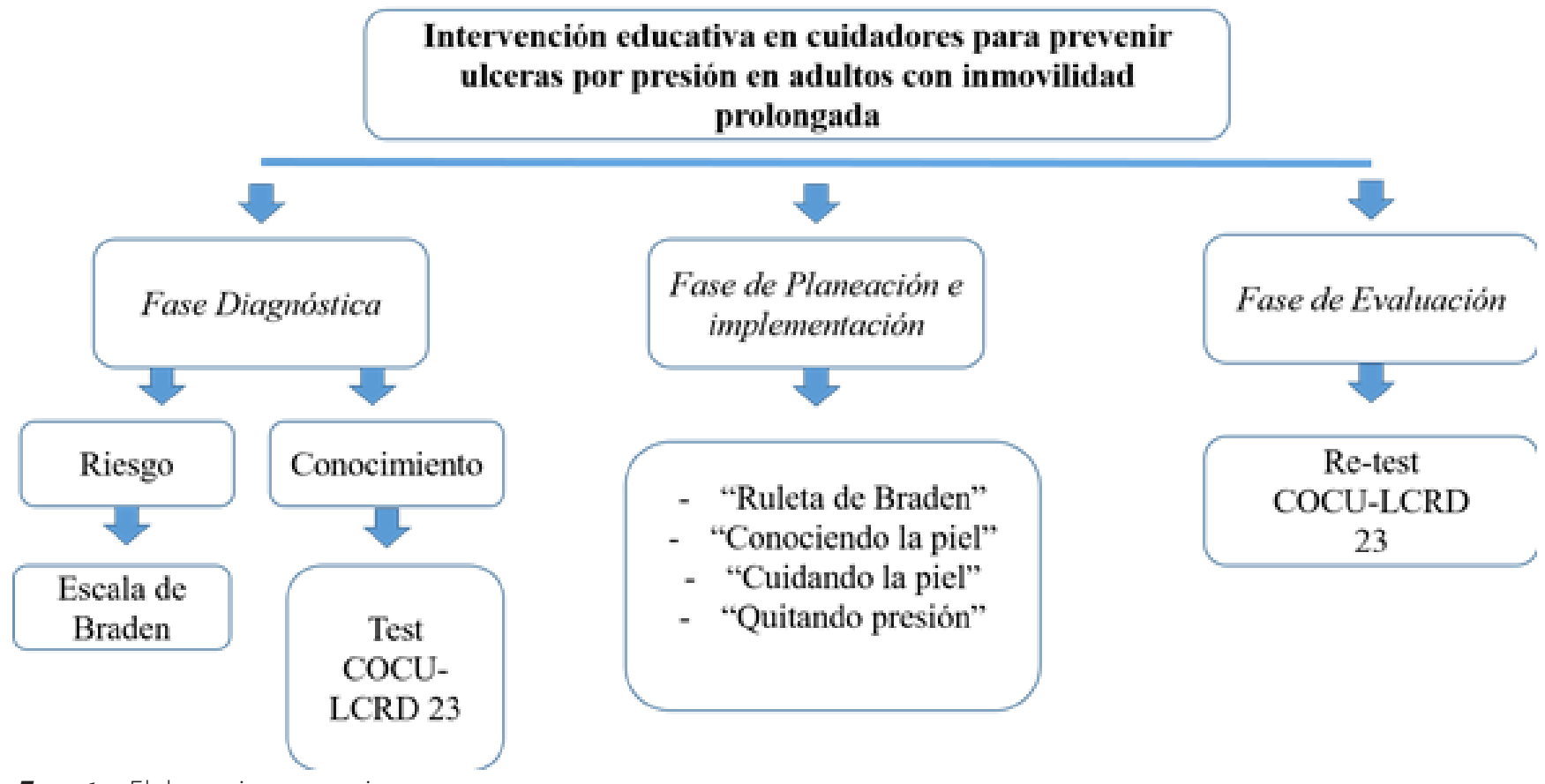

Fuente: Elaboracipon propia 
impartieron en forma de taller con el uso de materiales educativos visuales y validados. La primera sesión se denominó "Ruleta de Braden" se trata de una escala de valoración para cuidadores de pacientes con riesgo de padecer lesiones de la piel asociadas al reposo prolongado. La ruleta fue adaptada por los autores, retomados los parámetros de la escala original de Braden (18), de forma clara y sencilla sin puntos numéricos, sustituidos por códigos de colores que contienen el nivel de riesgo y las medidas para evitar la aparición de úlceras por presión (19). En la segunda sesión "conociendo la piel" mediante material informativo se explicó la importancia de mantener la integridad de la piel y los factores de riesgo. La tercera sesión se denominó "cuidando la piel", en la que se mostró a los cuidadores las zonas físicas de mayor presión y los estadios de la UPP, la cuarta sesión "quitando presión" tuvo el propósito de incrementar el nivel de conocimientos y habilidades para disminuir el riesgo de aparición de lesiones.

3. Fase de Evaluación. En esta fase se midió el efecto de la intervención educativa, con la aplicación del re-test, mediante el COCU- LCRD-23, posterior a la implementación de las sesiones educativas.

\section{RESULTADOS}

La muestra estuvo constituida por 15 participantes familiares que cumplieron con el rol de cuidadores primarios de pacientes con inmovilidad prolongada, de ambos sexos, donde predominó el sexo femenino (73\%), con un rango de edad de 25 a 58 años, en su mayoría tienen nivel de escolaridad primaria (47\%).

Para alcanzar el objetivo del programa educativo en la fase diagnostica y de evaluación, se midió el conocimiento antes y después de la intervención bajo un formato estadístico test, re-test para realizar las contrastaciones de medias aritméticas de muestras relacionadas. En la fase diagnostica (test) se obtuvo que $80 \%$ de los cuidadores no tenía conocimientos acerca de la prevención de úlceras por presión. En la fase de evaluación, se midió nuevamente el nivel de conocimientos (re-test), los hallazgos mostraron que posterior a la implementación del programa educativo, 100\% de los cuidadores incrementó el nivel de conocimiento, con un efecto positivo y estadísticamente significativo $(p=.000)$.

\section{DISCUSIÓN}

Tabla 1. Análisis descriptivo de la mediciones

\begin{tabular}{|l|l|l|}
\hline Medición & \multicolumn{1}{|c|}{$\begin{array}{c}\text { Promedio } \\
\text { aritmético }\end{array}$} & Desviación típica \\
\hline Test & 46.33 & \pm 2.31 \\
\hline Re-test & 55.45 & \pm 11.7 \\
\hline
\end{tabular}

Fuente: $C O C U$ - LRCD 23
Tabla 2. Prueba $t$ de Student para muestras relacionadas

\begin{tabular}{|c|l|l|l|l|}
\hline \multicolumn{1}{|c|}{} & gl & $\begin{array}{l}\text { Sig. (bila- } \\
\text { teral) }\end{array}$ & \multicolumn{2}{|c|}{$\begin{array}{c}\text { 95\% de intervalo } \\
\text { de confianza de la } \\
\text { diferencia }\end{array}$} \\
\hline 18.348 & 14 & .000 & $\begin{array}{l}\text { Inferior } \\
48.97\end{array}$ & $\begin{array}{l}\text { Superior } \\
61.94\end{array}$ \\
\hline
\end{tabular}

Fuente: COCU- LRCD 23

La investigación buscó determinar la eficacia de la intervención educativa sobre los conocimientos en la prevención de úlceras por presión en los cuidadores primarios de pacientes con inmovilidad prolongada. Los datos sociodemográficos reiteran que las mujeres son las encargadas de cumplir el rol de cuidador principal de sus familiares, reforzando con esto, los estándares establecidos socialmente en el que reconocen a la mujer como proveedora del cuidado dentro del núcleo familiar. Además, el predomino de escolaridad primaria como máxima en estas mujeres, disminuye la posibilidad de tener una relación laboral formal, lo cual las hace personas con mayor probabilidad para tomar este rol, debido la gran demanda de tiempo y cuidado por parte del paciente. Indagar y conocer los datos sociodemográficos de los cuidadores primarios, en términos de escolaridad y alfabetismo, ayuda a perfilar la estructuración de las herramientas a utilizar en los programas educativos.

Este estudio pone evidencia del conocimiento insuficiente de los cuidadores para la prevención de UPP, muestra la necesidad de la implementación de este programa para fortalecer en este grupo las habilidades encaminadas a la disminución de estas lesiones, resultados similares en otro estudio, donde se encontró una deficiencia en los conocimientos de los cuidadores y que al implementar su intervención tuvo un resultado significativo en la incrementación del nivel de los cuidadores en su totalidad (20).

Los hallazgos de este estudio, coincide con lo reportado por otros autores, donde al explicar el incremento de conocimientos posteriores a la implementación de una intervención, concluyeron que la educación para la salud, es efectiva para incrementar el conocimiento para la prevención de las UPP en los cuidadores ${ }^{(20,21)}$.

La contribución científica del estudio radicó en aportar una intervención de enfermería bajo un diseño experimental, situación que no es común en la literatura de la ciencia del cuidado, además de realizar el análisis estadístico. Aunado a lo anterior, no solo debe considerarse los promedios aritméticos, la significancia clínica de este trabajo denota una aportación bajo teoría fundamentada en la evidencia previamente publicada, lo que contribuye a los programas educativos de enfermería por la metodología de intervención utilizada. 


\section{CONCLUSIONES}

El resultado obtenido muestra eficacia estadísticamente significativa de las cuatro sesiones que conformaron el Programa Educativo para aumentar el nivel de conocimientos de los cuidadores para la prevención de UPP.

Limitaciones del estudio: El diseño cuasi-experimental por sí mismo representa una limitación al no contar con un grupo control, sin embargo, los mismos sujetos de estudio se comportaron como su propio control para realizar las contrastaciones estadísticas. El tamaño de la muestra restringió los grados de libertad, el número de sujetos incluidos a la intervención fue pequeño, no obstante, la distribución de los datos presentó normalidad, lo que permitió aplicar estadística paramétrica. Los resultados se consideran confiables a pesar de la muestra pequeña; durante el desarrollo de la investigación se conservó el poder estadístico dado que no hubo pérdida de sujetos de estudio.

\section{CONFLICTO DE INTERESES}

No existe conflicto de intereses entre los autores

\section{FINANCIAMIENTO}

Ninguno.

\section{REFERENCIAS BIBLIOGRÁFICAS}

1. Grupo Nacional para el Estudio y Asesoramiento en úlceras por Presión (GNEAUPP). [Internet] 2018[Consultado: 14 de septiembre de 2018]. Disponible en: https://gneaupp.info/ cuidados-de-enfermeria-en-la-prevencion-y-tratamientode-las-ulceras-por-presion/

2. Mijangos M, Puga C, Guillen L, Zúñiga I. El manejo de las ulceras por presión: intervenciones encaminadas a un oportuno manejo hospitalario. Evid Med Invest Salud [Internet] 2015[Acceso: 02 de septiembre 2018]; 8 (2): PP 77-83. Disponible en: http://www.medigraphic.com/pdfs/ evidencia/eo-2015/eo152e.pdf

3. Carrillo G, Sánchez B, Barrera L. Habilidad de cuidado de cuidadores familiares de niños con cáncer. Rev Salud Pública. [Internet] 2015[Acceso: 5 de agosto 2018]; 17 (3) 394-403. Disponible en: https://revistas.unal.edu.co/index. php/revsaludpublica/article/view/32408/62040

4. Godoy M, Huamaní B. Prevalencia de úlcera por presión en pacientes hospitalizados en una institución prestadora de servicios de salud. [Tesis de licenciatura]. Universidad Peruana Cayetano Heredia. Perú; 2017. Disponible en: http://repositorio.upch.edu.pe/bitstream/ handle/upch/1492/Prevalencia_GodoyGalindo_Melissa. pdf? sequence $=1 \&$ is Allowed $=y$

5. Rivas JC, Ostiguín RM. Cuidador: ¿concepto operativo - preludio teórico? Rev Enferm Univ ENEO-UNAM [Internet] 2011 (8) 1: [Acceso: 20 marzo 2018]; Disponible en: $\quad$ http://www.scielo.org.mx/scielo.php?script=sci_ arttext\&pid $=$ S1665-70632011000100007

6. Maynard R, Barthley L, Hodelín H, Santiago D, Michel G. Capacitación para cuidadores primarios de personas adultas mayores dependientes desde la educación popular. Rev. inf. cient. [Internet] 2016[Acceso: 13 marzo de 2019]; 95 (2). Disponible en: http://www.revinfcientifica.sld.cu/index.php/ ric/article/view/101

7. Mayorga J. Efectividad de una intervención educativa en cuidadores primarios de pacientes con ulceras por presión en reposo prolongado hospitalizados en el HGZ NO. 1 del IMSS, delegación Aguascalientes de diciembre 2015 a febrero 2016 [Tesis de especialidad]. [Aguascalientes]: Universidad Autónoma de Aguascalientes; 2016. Disponible en: $\quad$ http://bdigital.dgse.uaa.mx:8080/xmlui/bitstream/ handle/11317/482/408653. pdf? sequence=1\&isAllowed =y

8. Wandosell J, Salgado Á, Moreno M, Rodríguez S, Paz M, Mañá A, et al. Efectividad de una intervención formativa en prevención de úlceras por presión en una unidad de cuidados intensivos quirúrgica: un estudio cuasi experimental. Gerokomos [Internet] 2012[Acceso: 14 de marzo de 2019]; 23 (3): 128-131. Disponible en: http:// scielo.isciii.es/scielo.php?script=sci_arttext \&pid=\$1134 928X2012000300007\&lng=es. http://dx.doi.org/10.4321/ S1134-928X2012000300007

9. Hernández N, Barragán J, Moreno C. Intervención de enfermería para el bienestar de cuidadores de personas en cuidado domiciliario. Rev Cuid. [Internet] 2018[Acceso: 14 de febrero de 2019]; 9(1): 2045-58. Disponible en: http://dx.doi. org/10.15649/cuidarte.v9i1.479

10. Rodríguez M, Díaz J. Herramientas para cuidadores de pacientes con heridas crónicas Serie Documentos Técnicos GNEAUPP no 13. 2016. [Internet] 2016 [Consultado: 14 de octubre de 2018]. Disponible en: https://gneaupp.info/ wp-content/uploads/2016/05/Doc-Posicionamiento-14Herramientas-para-cuidadoras.pdf

11. GNEAUPP. Guía para la prevención y manejo de las UPP y heridas crónicas. [Internet] 2015 [Acceso: 13 marzo 2019]. Disponible en: http://gneaupp.info/guia-para-laprevencion-y-manejo-de-las-upp-y-heridas-cronicas/

12. Martin D, Albensi L, Van S, Froese M, Montgomery M, Lam M, et al. Healthy Skin Wins: A Glowing Pressure Ulcer Prevention Program That Can Guide Evidence-Based Practice. Worldviews Evid Based Nurs. [Internet] 2017[Acceso: 
7 de marzo de 2019];14(6):473-483. Disponible en: https:// sigmapubs.onlinelibrary.wiley.com/doi/epdf/10.1111/ wvn.12242

13. Martínez J, Del Pino R. Enfermería en Atención Primaria. 1era Ed. Madrid - España: Difusión Avances de Enfermería; 2006. 197p.

14. Arboledas J, Pancorbo P. Cuestionario de conocimientos de cuidadores familiares sobre la prevención de úlceras por presión y lesiones cutáneas relacionadas con la dependencia: desarrollo y validación. Gerokomos. [Internet] 2016[Acceso: 28 enero 2018]. 27 (2): 73-79. Disponible en: http://scielo.isciii.es/scielo.php?script=sci_ arttext\&pid=\$1134928X2016000200008\&lng=s

15. Polit D, Beck C. Investigación en enfermería: fundamentos para el uso de la evidencia en la práctica de la enfermería. 9 th Ed. North American: Wolters Kluwer; 2017.

16. Diario Oficial de la Federación. Reglamento de la Ley General de Salud en Materia de Investigación para la Salud. México; 2014. [Consultado enero de 2018]. Disponible en: http://www.salud.gob.mx/unidades/cdi/nom/compi/rlgsmis. html

17. Nieto J, Esteban M, Tejerina M, Abad M. Psicología para Ciencias de la Salud. Estudio del comportamiento humano ante la enfermedad. 1era Ed. España: McGraw- HillInteramericana de España; 2015. 24p.

18. Larrea B, Vazquez M, Labiano J. Evaluación del impacto de una intervención de enfermería en la prevención y tratamiento de las úlceras por presión. Gerokomos. [Internet] 2015[Acceso: 18 febrero 2018]; 26(3):115-119

Disponible en: http://scielo.isciii.es/pdf/geroko/v26n3/09_ helcos4.pdf

19. García F, Soldevilla J, Pancorbo P, Verdú J, López P, Rodríguez M. Clasificación-Categorización de las lesiones relacionadas con la dependencia. Serie Documentos Técnicos GNEAUPP no II. [Internet] 2014[Consultado: 19 de febrero de 2018]. Disponible en: https://gneaupp.info/ clasificacion-categorizacion-de-las-lesiones-relacionadascon-la-dependencia-2/

20. Arpasi O. Efectividad del programa educativo "Cuidando con amor" en los conocimientos, actitudes y prácticas sobre prevención de úlceras por presión de los cuidadores de pacientes adultos mayores del Hospital San Isidro Labrador EsSalud, Lima, Perú. Rev Cient de Ciencias de la Salud [Internet] 2013 6(2): [Acceso: 20 marzo 2018]; Disponible en: https://revistas.upeu.edu.pe/index.php/rc_salud/article/ view/1028

21. Soto O, Barrios S. Caracterización de la salud, 